\title{
Performance of Low-voltage Electron Microscope with New Aberration Correction System and Cold Field Emission Gun
}

\author{
T. Sasaki ${ }^{*, * *}$, H. Sawada ${ }^{*, * *}$, T. Nakamichi, ${ }^{* *}$, F. Hosokawa ${ }^{* *}$, K. Omoto ${ }^{* * *}$, T. Tomita ${ }^{* * *}$, \\ T. Kaneyama*, ${ }^{* * *}$ Y. Kondo ${ }^{* *}$, K. Kimoto ${ }^{* * * *}$, K. Suenaga*,* \\ *Japan Science and Technology Agency, CREST, 5, Sanbancho, Chiyoda-ku, Tokyo 102-0075, \\ Japan \\ ${ }^{* *}$ JEOL Ltd., 3-1-2 Musashino, Akishima Tokyo 196-8558, Japan \\ *** National Institute for Materials Science, 1-1, Namiki, Tsukuba, Ibaraki, 305-0044, Japan \\ ${ }^{* * * *}$ Nanotube Research Center, National Institute of Advanced Industrial Science and Technology \\ (AIST), Central 5, 1-1-1 Higashi, Tsukuba, Ibaraki 305-8565, Japan
}

For characterization of soft materials, which contain light elements, it is of great effective to apply lower accelerating voltage in order to observe or analyze them at high resolution free from radiation damage originated from a probing electron beam. Thus, we have been developing a low-voltage electron microscope [1] equipped with a double spherical aberration correction system for scanning transmission electron microscopy (STEM) and transmission electron microscopy (TEM) designed by us and a cold field emission gun (CFEG). This paper reports the results of performance test for this microscope.

Figure 1 (a) shows the developed low-voltage electron microscope. Attainable range of accelerating voltage is from 30 to $60 \mathrm{kV}$. We employed a new aberration correction system for compensation of spherical aberration, which is a triple dodecapole system (DELTA-corrector) [2]. The DELTAcorrector can compensate six-fold astigmatism $\left(\mathrm{A}_{6}\right)$, which cannot be compensated by the double hexapole (or dodecapole) systems [3,4]. We developed dedicated software for compensating aberrations up to 5-th order. Residual aberrations in STEM and TEM are calculated by a segmental Ronchigram autocorrelation function matrix (SRAM) method [5] and by a diffractogram tableau method [6], respectively. These are automatically compensated through the software assisted feedback loop. Figure 1 (b) shows one of the typical correction results with the DELTA-corrector. In this operation GUI, buttons for automated aberration correction are arranged in the left side (area 1) and the diffractogram tableau is shown in the middle area (2). The table shown in (c) represents results calculated by this system. The $\mathrm{A}_{6}$ is well compensated in the table. Figure 2 shows a highangle annular dark field (HAADF) image of $\mathrm{Si}<110>$ taken at $60 \mathrm{kV} .0 .14-\mathrm{nm}$-separated silicon-tosilicon dumbbells are clearly resolved, and its power spectrum unambiguously indicates information of $0.11 \mathrm{~nm}$. These were not achieved without correction of $\mathrm{A}_{6}$. Figure 3 (a) and (b) show a highresolution TEM (HRTEM) image and its power spectrum. The lattice information at $0.12 \mathrm{~nm}$ is achieved in both the image and the power spectrum. Although a Young's fringe method does not provide accurate quantification of the information limit, it is worthy to mention the results of the Young's fringe test as a qualitative estimation [7]. Young's fringe patterns, which are recorded from a thick sample of gold grating on carbon amorphous, are observed up to $0.083 \mathrm{~nm}$ as shown in Fig. 3 (c). These results demonstrate the capability of this low-voltage electron microscope.

\section{References}

[1] K. Suenaga et al, to be published.

[2] H. Sawada et al., these proceedings.

[3] H. Rose, Optik 85 (1990) 19.

[4] F. Hosokawa et al., IMC 16 proceedings (2006) 582.

[5] H. Sawada et al., Ultramicroscopy 108 (2008) 1467. 
[6] F. Zemlin et al., Ultramicroscopy 3 (1978) 49.

[7] J. Barthel and A. Thust, Phys. Rev. Lett. 101 (2008) 200801.

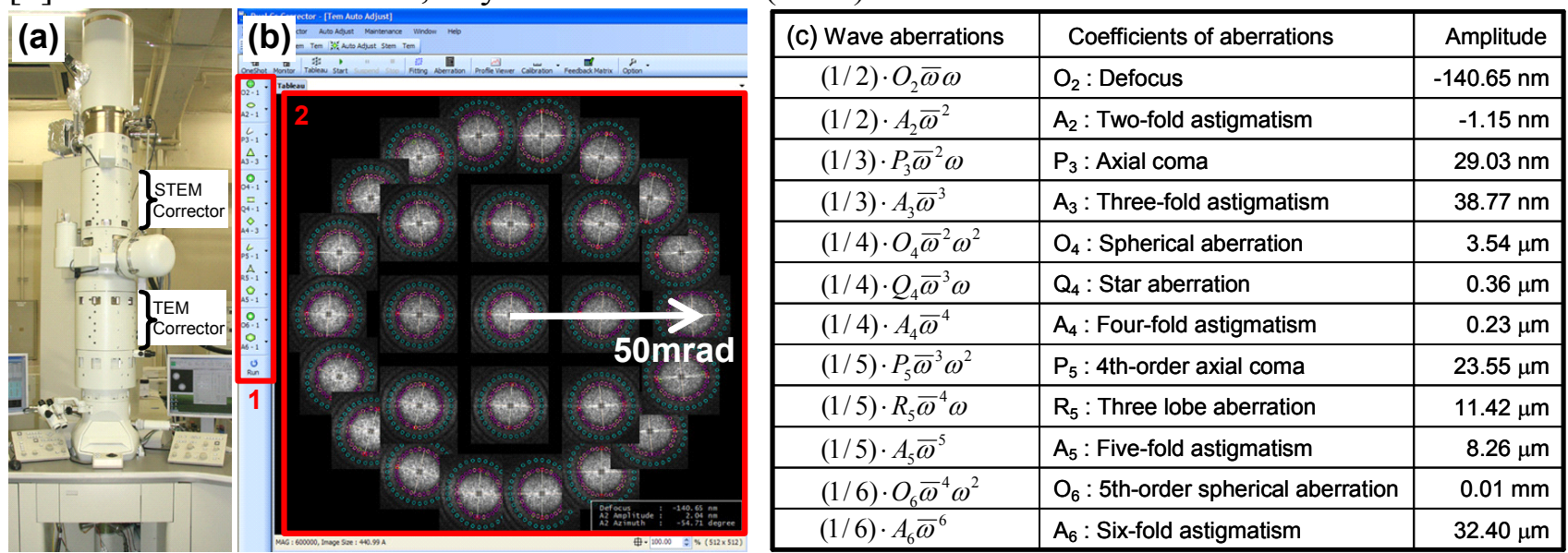

Fig.1 (a) Developed low-voltage electron microscope equipped with a CFEG and aberration correction systems for STEM and TEM (b) a snapshot of the TEM DELTA-corrector operation system. (c) Typical results of aberration correction shows that six-fold astigmatism is well compensated as well as other aberrations.
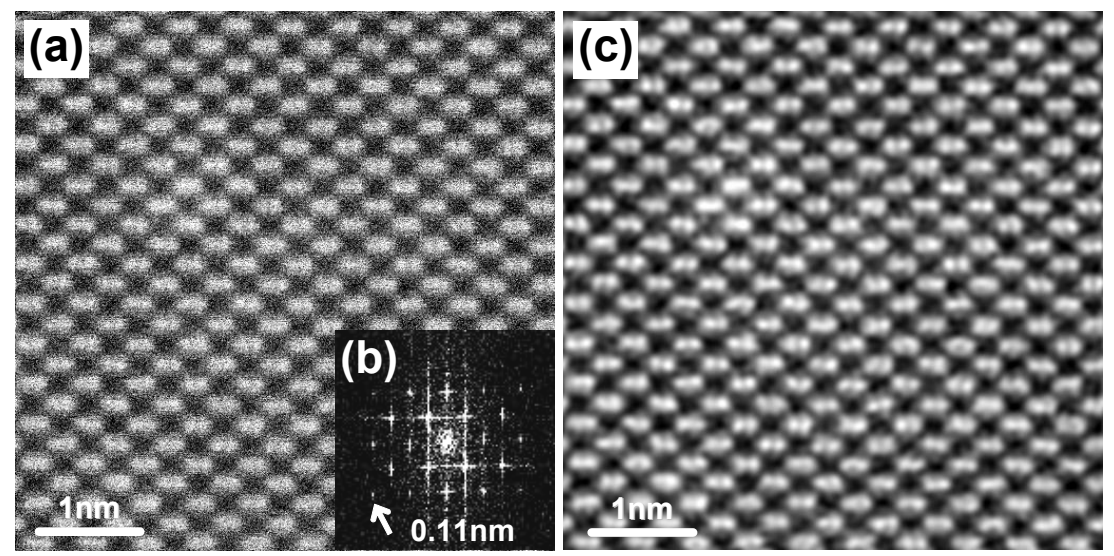

Fig.2 (a) HAADF image (raw data) of $\mathrm{Si}<110>$ at $60 \mathrm{kV}$, (b) its power spectrum and (c) low-pass filtered image are shown.
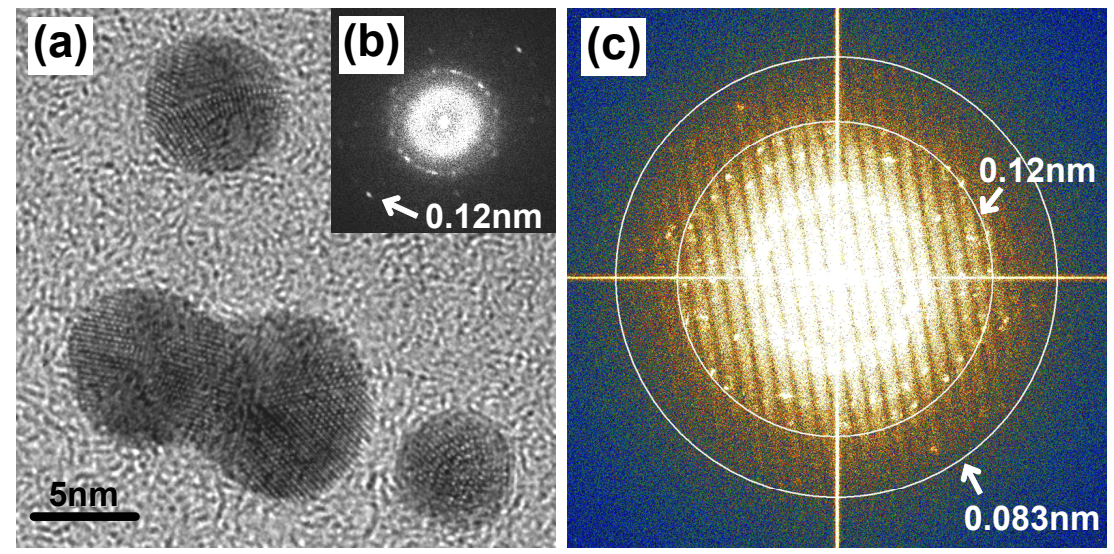

Fig.3 (a) HRTEM image (raw data) of gold particles onto Ge amorphous at $60 \mathrm{kV}$ and (b) its power spectrum. (c) Young's fringe patterns recorded from a thick gold grating at $60 \mathrm{kV}$. 